\title{
Conceptual Design of the EU DEMO EC-System: Main Developments and R\&D Achievements
}

G. Granucci ${ }^{1}$, G. Aiello ${ }^{2}$, S. Alberti ${ }^{3}$, K. A. Avramidis ${ }^{2}$, F. Braunmüller ${ }^{3}$, A.Bruschi ${ }^{1}$, J. Chelis $^{4}$, J. Franck ${ }^{2}$, L.Figini ${ }^{1}$, G. Gantenbein ${ }^{2}$, S. Garavaglia ${ }^{1}$, G. Grossetti ${ }^{2}$, S. Illy ${ }^{2}$, Z. Ioannidis $^{2}$, J. Jelonnek ${ }^{2}$, P. Kalaria ${ }^{2}$, G. Latsas ${ }^{4}$, A. Moro ${ }^{1}$, I. Gr. Pagonakis ${ }^{2}$, D. Peponis ${ }^{4}$, E. Poli ${ }^{5}$, N. Rispoli ${ }^{1}$, T. Rzesnicki ${ }^{2}$, T. Scherer ${ }^{2}$, D. Strauss ${ }^{2}$, M. Thumm ${ }^{2}$, I. Tigelis ${ }^{4}$, C.Tsironis ${ }^{4}$, C. $\mathrm{Wu}^{2}$, T. Franke ${ }^{5,6}$ and M. Q. Tran $^{3}$

${ }^{1}$ Institute of Plasma Physics "P.Caldirola”, National Research Council of Italy, Milan, Italy

${ }^{2}$ Karlsruhe Institute of Technology (KIT), D-76131 Karlsruhe, Germany

${ }^{3}$ Swiss Plasma Center, EPFL, CH-1015 Lausanne, Switzerland

${ }^{4}$ NCSRD/NKUA, Faculty of Physics, \& National Technical University of Athens, School of Electrical and Computer Engineering, Athens, (Greece)

${ }^{5}$ Max-Planck-Institut für Plasmaphysik, Boltzmannstr. 2, D-85748 Garching, Germany

${ }^{6}$ EUROfusion Consortium, Boltzmannstr. 2, D-85748 Garching, Germany

E-mail contact of main author: granucci@ifp.cnr.it

\begin{abstract}
For the development of a DEMOnstration Fusion Power Plant the design of auxiliary heating systems is a key activity in order to achieve controlled burning plasma. The present heating mix considers Electron Cyclotron Resonance Heating (ECRH), Neutral Beam Injection (NBI) and Ion Cyclotron Resonance Heating (ICRH) with a target power to the plasma of about 50MW for each system. The main tasks assigned to the EC system are plasma breakdown and assisted start-up, heating to L-H transition and plasma current ramp up to burn, MHD stability control and assistance in plasma current ramp down. The consequent requirements are used for the conceptual design of the EC system, from the RF source to the launcher, with an extensive R\&D program focused on relevant technologies to be developed. Gyrotron: the R\&D and Advanced Developments on EC RF sources are targeting for gyrotrons operating at $240 \mathrm{GHz}$, considered as optimum EC Current Drive frequency in case of higher magnetic field than for the 2015 EU DEMO1 baseline. Multi-purpose (multi-frequency) and frequency step-tunable gyrotrons are under investigation to increase the flexibility of the system. As main targets an output power of significantly above $1 \mathrm{MW}$ (target: $2 \mathrm{MW}$ ) and a total efficiency higher than $60 \%$ are set. The principle feasibility at limits of a $236 \mathrm{GHz}$, conventional-cavity and, alternatively, of a $238 \mathrm{GHz}$ coaxial-cavity gyrotron are under investigation together with the development of a synthetic diamond Brewster-angle window technology. Advanced developments are on-going in the field of multi-stage depressed collector technologies. Transmission Line (TL): Different TL options are under investigation and a preliminary study of an evacuated quasi-optical multiple-beam TL, considered for a hybrid solution, is presented and discussed in terms of layout, dimensions and theoretical losses. Launcher: Remote Steering Antennas have been considered as a possible launcher solution especially under the constraints to avoid movable mirrors close to the plasma. With dedicated beam tracing calculations, the deposition locations coverage and the wave absorption efficiency have been investigated, considering a selection of frequencies, injection angles and launching points. An option for the EC system structure is proposed in clusters, in order to allow the necessary redundancy and flexibility to guarantee the required EC power in the different phases of the plasma pulse. Number and composition of the clusters are analysed to have high availability and therefore maximum reliability with a minimum number of components.
\end{abstract}

\section{Introduction}

For the development of a DEMOnstration Fusion Power Plant, the design of auxiliary heating systems is a key activity in order to achieve controlled burning plasma. At present the considered heating systems [1] are Electron Cyclotron (EC), Neutral Beam Injection (NBI) [2] and Ion Cyclotron (IC) [3] heating with a design target power to the plasma of about 50 MW for each system. Present simulations of various scenarios together with extrapolation 
from present tokamaks generation have been used to define the main tasks assigned to EC system design, which must be assured during the entire pulse. These requirements are used as inputs for the conceptual design of EC system, from the RF source to the launcher, with an extensive R\&D program focusing on relevant technologies not yet available and to be developed in view of DEMO. The main physical tasks are listed in the table 1 collecting what has been used in the performed simulations of the possible scenarios [4], [5], [6]. The described design is based on the pulsed EU DEMO1 2015 [7], where Electron Cyclotron Current Drive (ECCD) does not have a major role for pulse sustainment, but essentially for Neoclassical Tearing Mode (NTM) control during plasma flat top. The total powers for Ramp up and Ramp down, calculated in [1,4], are larger and the value of $50 \mathrm{MW}$ in the table 1 assumed that the whole design value (50MW) for Electron Cyclotron Waveguide (ECW) will be allocated to these functions. With regards to heating and current drive frequencies, the DEMO baseline is not decided yet since presently the activities in the EU are still in the preconceptual phases and various options are still under consideration. Based on the current assumptions for the EU DEMO1 2015 baseline, the two frequencies $170 \mathrm{GHz}$ for heating and $204 \mathrm{GHz}$ for current drive have been selected as base for the system design presented in this paper. The frequency $204 \mathrm{GHz}$ corresponds to a moderate upshift of 1.2 for the current drive function. The couple $(170 \mathrm{GHz}, 204 \mathrm{GHz})$ was selected to satisfy the natural resonance criteria of a diamond window. Nevertheless, the gyrotron R\&D is continuing to focus on 240 $\mathrm{GHz}$ as possible EC frequency for a future DEMO or Fusion Power Plant [8] with a higher toroidal magnetic field [9] as the RF source shall be prepared for challenging heating and, mainly, current drive scenarios. The final choice will be done once the EU DEMO design phase will be completed.

Table 1. Main EU DEMO EC tasks with corresponding power required and deposition location, assuming the design value of $50 \mathrm{MW}$. For all these functions, $100 \%$ reliability is expected.

\begin{tabular}{|l|c|c|c|}
\hline \multicolumn{1}{|c|}{ EC Task } & Power $[\mathbf{M W}]$ & Localization $[\boldsymbol{\rho}]$ & Mode \\
\hline Assisted Breakdown & $6-10$ & $<0.3$ & Heating \\
\hline Ramp up and L-H transition & 50 & $<0.3$ & Heating/CD \\
\hline Main heating & 50 & $<0.3$ & Heating/CD \\
\hline Sawtooth control & $2-10$ & $<0.3$ & CD \\
\hline NTM control $(\mathrm{q}=2 ; \mathrm{q}=3 / 2)$ & $10-15$ & $0.85 ; 0.75$ & CD \\
\hline Ramp down & 40 & $0.3-0.5$ & Heating \\
\hline
\end{tabular}

\section{Gyrotron R\&D and Advanced Developments}

The present EC conceptual design focuses on the EU DEMO1 2015 baseline for a pulsed machine with aspect ratio $\mathrm{AR}=3.1$ and $\mathrm{B}_{\mathrm{T}}=5.7 \mathrm{~T}$. Assuming maximum compatibility with ITER the heating frequency will be at $170 \mathrm{GHz}$. Taking into account a moderate upshift factor of 1.2, the corresponding optimum Electron Cyclotron Current Drive (ECCD) frequency will be $204 \mathrm{GHz}$. While the EC conceptual design is following this new baseline for a 2 hours pulsed machine, the gyrotron $R \& D$ and Advanced Developments are focusing on a possible operation up to $240 \mathrm{GHz}$, originally considered as a compromise between the optimum ECCD frequency for the 2012 EU DEMO1 baseline and a considerably major step for high frequency, high power (2MW) gyrotron. Hence, the continuation of this target is anticipating the possible long term options for a steady state DEMO (EU DEMO2 2015), with EC operation at higher toroidal fields at higher upshift of the frequency and with plasma parameters and launching angles requiring frequencies significantly above $200 \mathrm{GHz}$. 
Additionally, "multi-purpose (multi-frequency)" and "frequency step-tunable" operations are under investigation. "Multi-purpose" operation is targeting for possible RF output frequencies corresponding to multiples of the half-wavelength of the single-disc RF diamond window ( $\sim 34 \mathrm{GHz}$ steps for a window thickness of $\sim 1.85 \mathrm{~mm}$ ) allowing EC operation at different magnetic field configurations (slowly-varying) and at different discrete frequencies for heating and current drive (e.g. 136/170/204/238GHz). "Frequency step-tunability" is targeting for the fast stepping (in seconds) of the operating frequency. Steps of $2-3 \mathrm{GHz}$ shall be possible using a broadband RF output window technology (Brewster angle window). That will allow the fine tuning of the deposition location. Most important in the realization of the Chemical Vapor Deposition (CVD) diamond-disc Brewster-angle window technology is, firstly, to find a proper solution for the production of large diamond discs windows, secondly, to realize suitable assembly technologies for merging the diamond discs window with the copper cuff, and, thirdly, to achieve a sufficient cooling during operation. Finally, in the project, it is considered to push the total efficiency of gyrotrons to above $60 \%$ by using multistage depressed collectors (MSDC), compared to the presently achieved value in the range of $50 \%$. Different MSDC concepts are under investigation.

Two different concepts are under investigation to check the principle feasibility for a $2 \mathrm{MW}$ gyrotron operating at $240 \mathrm{GHz}$. Those are the conventional-cavity technology, and, alternatively, the EU coaxial-cavity technology [10].
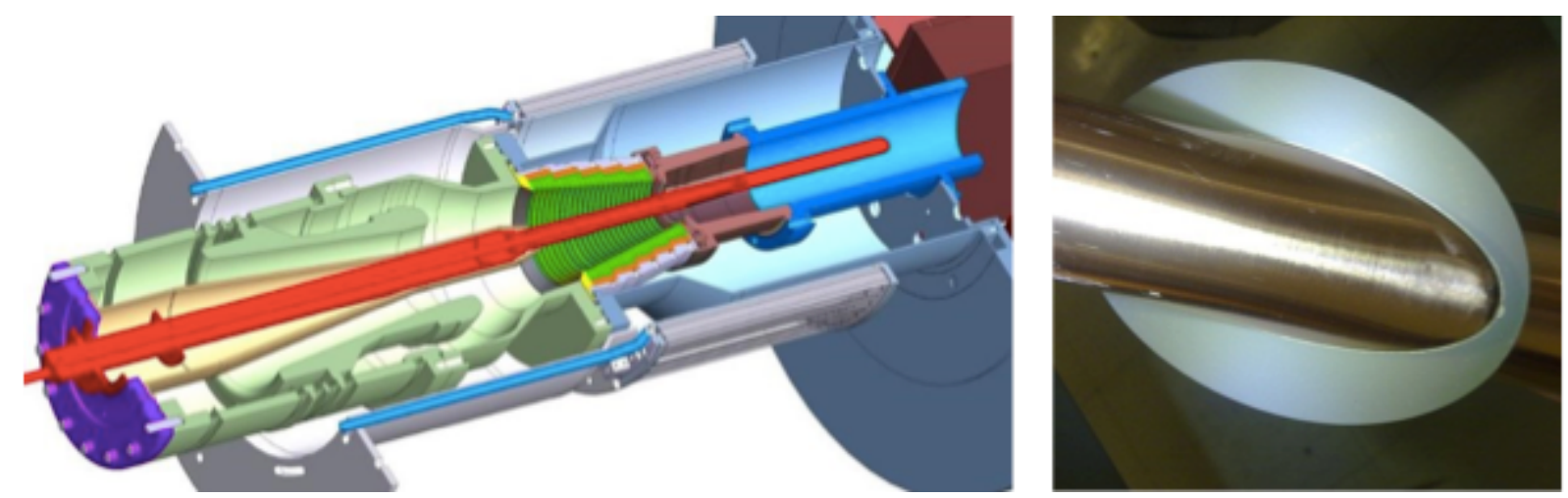

Figure 1. Left: Implementation of the insert into a coaxial-cavity gyrotron. Right: CVD-diamond disc mounted into a circular waveguide (diameter: $50 \mathrm{~mm}$ ) at the Brewster-angle of $67.2^{\circ}$.

The figure 1 (left) is showing the relevant part of a coaxial-cavity design. The coaxial insert is in red. The on-going research is focusing on the maximum achievable output power and efficiency versus operating stability. Physical and technical restrictions considered in the designs are: a spread of the electron guiding centres $\leq \lambda / 5$; an emitter current density $\leq 4 \mathrm{~A} / \mathrm{cm}^{2}$; an ohmic loading on the cavity wall $\leq 2 \mathrm{~kW} / \mathrm{cm}^{2}$. In [11] a generic mode selection strategy for highly overmoded multi-frequency gyrotrons with a quasi-optical output coupler and a CVD diamond single-disc window is presented in a very general form. The strategy bases on an expedient rating scheme for different series of operating modes. It is shown that a simple relation can be derived which provides a proper indication whether a certain mode lies within a multi-frequency suitable series. Based on these considerations, one finds that a coaxial-cavity three-frequency gyrotron with the $\mathrm{TE}_{49,29}$-mode at $237.5 \mathrm{GHz}$ is expected to retain sufficiently low internal losses if operated at the allied modes $\mathrm{TE}_{42,25}$ at $203.6 \mathrm{GHz}$ and $\mathrm{TE}_{35,31}$ at $170.0 \mathrm{GHz}$. From the simulation an output power of about $2 \mathrm{MW}$ seems to be possible. One of the most important challenges of the coaxial-cavity design is that the coaxialinsert might be slightly misaligned with respect to the cavity wall, due to manufacturing tolerances. Depending on how large this misalignment actually is, it will lead to a drop in interaction efficiency, mode purity and/or mode stability, and thus in output power and 
reliability. Since gyrotrons are welded and evacuated after assembly, one might be unable to fully compensate such an internal misalignment after bake-out in case it turns out to be critical. Therefore, one needs to define misalignment tolerances based on realistic simulations beforehand. In [12] possible effects of the insert misalignment on the electron beam and the electromagnetic modes, and how to treat those effects numerically in interaction simulations are shown. The theoretical considerations are supported by experimental campaigns to verify the superior performance of the coaxial-cavity technology [13 14]. The further development of a longer-pulse $2 \mathrm{MW} 170 \mathrm{GHz}$ gyrotron is in progress. A new magnetron injection gun (MIG) which satisfies all design criteria [15] for the suppression of the harmful electron trapping is under manufacturing. In the new MIG design, an advanced type of emitter ring [16] is used in order to decrease the sensitivity of the manufacturing tolerances on the gyrotron performance. In parallel, an innovative type of electron gun, which is more dedicated for coaxial gyrotron technology, has been designed [17] and currently is under manufacturing at KIT. Furthermore, an advanced water cooling system for the beam tunnel, cavity and launcher has been designed for the extension of the pulse length towards the 1s keeping the modularity of the gyrotron.

With regards to the conventional-cavity technology a generalized, systematic cavity design approach has been proposed and implemented for a $\mathrm{TE}_{43,15}$-mode cavity at $236 \mathrm{GHz}$ (see [18]). Based on this, optimum operating parameters of the gyrotron are shown. Using the EURICIDE code [19], a time-domain self-consistent simulation has been performed to verify the interaction performance. In total, 99 competing modes have been taken into account. Stable RF output of about $920 \mathrm{~kW}$ has been achieved with an interaction efficiency of $36 \%$. With the suggested mode series, multi-frequency operation of the EU DEMO gyrotron has been estimated at $170 \mathrm{GHz} / 203 \mathrm{GHz} / 236 \mathrm{GHz}$ and $269 \mathrm{GHz}$. In [20] the possibility for fast frequency step-tunability in leaps of $2-3 \mathrm{GHz}$ steps is presented for the $236 \mathrm{GHz}$ conventional-cavity gyrotron. The selection of modes for the frequency-tunable gyrotron is restricted by the requirements that they should have large coupling, suitable frequencies, and their caustic radius should be nearly identical in order to have the same quasi-optical launcher design for all the selected modes. Efficient MW-level operation within a $\pm 10 \mathrm{GHz}$ frequency range is demonstrated for the selected modes. The simulation results support very high conversion efficiency for all selected modes with the designed hybrid-type launcher of the quasi-optical output coupler. Here, it has to be considered that the gyrotron operating frequency primarily depends on the operating magnetic field. The time required to move from one frequency to its neighbouring one is governed by the rate of change in the cavity magnetic field, hence, it is limited by the tuning capability of the superconducting (SC) magnet. So far, it is estimated that it will be possible to do a frequency step in the range of seconds.

Apart from an advanced fast-switching SC magnet, a broadband output window is necessary for a frequency-tunable gyrotron. The transmission properties of a conventional single-disc window allow gyrotrons only to have operating frequencies at $f_{N}=\left(c\left(2 \cdot d \cdot \sqrt{ }\left(\varepsilon_{\mathrm{r}}\right)\right) \cdot N=f_{l} \cdot N\right)$ within an $\pm 1 \mathrm{GHz}$ frequency range. Here, $d$ is the window thickness, $\varepsilon_{\mathrm{r}}$ is the relative permittivity of the synthetic diamond material and $N$ is an integer number. For a standard RF window thickness (ranging from $1.50 \mathrm{~mm}$ to $2.25 \mathrm{~mm}$ typically) of a CVD-diamond disc $\left(\varepsilon_{\mathrm{r}}=5.67\right)$, the corresponding fundamental frequency $\left(\mathrm{f}_{1}\right)$ ranges from $\sim 30 \mathrm{GHz}$ to $40 \mathrm{GHz}$, which does not support step-frequency tunability [21]. The Brewster-angle window, which has a wide transmission band, is a favourable option to achieve frequency step-frequency tunability. Figure 1 (right) shows a Brewster angle window disc mounted into a circular waveguide of $50 \mathrm{~mm}$ diameter; the Brewster-angle is $67.2^{\circ}$.

A first successful implementation into a megawatt-class D-band $(111.6 \mathrm{GHz}-165.7 \mathrm{GHz})$ short-pulse prototype gyrotron operating at 10 different frequency steps and related results 
from experiments at short pulses (ms) have been reported in $[22,23]$. The fundamental issue in the Brewster-angle technology is the production of a diamond disc with a sufficiently large axis. Ideally, a typical gyrotron window would require an aperture of $80 \mathrm{~mm}$. That results in the need for a diamond disc with a minimum large diameter of $220 \mathrm{~mm}$. Today, a manufacturing technology to produce such large diamond discs does not exist. Within the EUROfusion project it was thus decided to, firstly, set a more realistic target for this project. Hence, a waveguide with an aperture of $63.5 \mathrm{~mm}$ is considered. As possible future production technology the joining of a mosaic disc is under investigation. The joining itself might be performed joining the disc parts together by a localized CVD diamond growth process.

Beside an output power of significantly above $1 \mathrm{MW}$ (target $2 \mathrm{MW}$ ), to minimize the recirculating power, a total efficiency of above $60 \%$ are required for a future EU DEMO gyrotron. Single-stage depressed collectors will allow a maximum efficiency of electron energy recuperation of maximum $60 \%$. Assuming an electronic efficiency of $35 \%$ for the gyrotron interaction, the total efficiency is limited to $\sim 50 \%$. Hence MSDCs are mandatorily required for future fusion gyrotrons. Different concepts for MSDC are under investigation. Three major items will have to be overcome: the large spread of the kinetic energy of the electrons after the gyrotron interaction; the large transversal energy of the electrons and the large axial magnetic field of the SC magnet at the gyrotron collector. First approaches for MSDCs, which can be found in the literature, have not been successful [24-27]. Major reason is the generation of secondary electrons, which deteriorate the performance of the gyrotron. Another very promising approach has been published in [28]. This second concept uses the $\mathrm{E} \times \mathrm{B}$ drift to sort and drift electrons towards the electrodes according to their initial velocities. In order to apply the proposed design approach to conventional gyrotrons, the transformation of the cylindrical hollow electron beam to one or more sheet beams is proposed using appropriate electrostatic and magnetostatic fields. Several conceptual designs an MSDC system based on that idea are proposed in [29] and [30] which results in a collector efficiency up to $86 \%$.

\section{EC System Conceptual Design}

The conceptual design of the EC system is based on subsystems performances chosen to fulfil the tasks reported in table 1 together with the constrains typical of a reactor producing electricity, as it is DEMO. The ideal required overall reliability to deliver 50MW with EC system is $100 \%$ and can be obtained using redundancy and subsystems with high enough singular reliability. The system will be based therefore on a strong modularity and on the possibility to substitute each failing subunit with one in standby. The basic architecture is a cluster of $n$ sources (gyrotrons) fed by only one high voltage power supply for the cathode current and one anode power supply for each gyrotron. The $n$ microwave beams are transmitted by single multi-beam line (considered a unique subsystem, even if composed by separate waveguides) and delivered to a single launcher composed by $n$ independent antennas hosted in one port. An exception can be considered for the cluster dedicated to NTM stabilization that feeds two different launchers (4 beams each) potentially located in two different (vertical) ports. Using this modularity, the system reliability (defined as the ability of an item to perform a required function, under given environmental and operational conditions and for a stated period of time [31, 32]) has been calculated, assuming $98 \%$ of reliability for the gyrotron, $99.9 \%$ for the transmission line and $99.9 \%$ for the launcher. As figure of merit we use the Mean Time Between Failures (MTBF) that must be as high as possible. The EU DEMO pulse length being 2 hours, 1000 pulses between two (major) faults of EC system lead to $2000 \mathrm{~h}$ for the MTBF, which is equivalent to $99.9 \%$ of reliability. The analysis [33] allowed the selection of proper optimized number of elements of each cluster 
$(n=8)$ and the total number of cluster: $4+1$ for main tasks and one for NTM stabilization. The chosen values are the result of an optimization processes that minimize the total number of gyrotrons/elements and maximize the number of beams on each mirror ensuring the highest possible MTBF [32]. In this scheme one line in each operating cluster is in stand-by and one whole cluster is also in stand-by, ready to substitute a faulty cluster. Based on this analysis, to insure $>99.9 \%$ reliability, the total number of $2 \mathrm{MW}$ gyrotrons considered in the system is therefore 40, 28 of them in operation to deliver 50MW to the plasma, assuming $10 \%$ of losses in the transmission of the power for a distance for $100-150 \mathrm{~m}$ (see table 2).

Source: We consider a gyrotron capable to operate at (two) different frequencies $(170 \mathrm{GHz}$ and $204 \mathrm{GHz}$ ) with the same power level (2MW), same efficiency (60\%), Gaussian output content (98\%) and reliability (98\%). These numbers are general requirements that we assume technologically mature at DEMO construction, even not already possible for the present generation of the tubes developed for ITER. The two frequencies (see figure 2) will be used to fulfil pure ECH task $(170 \mathrm{GHz})$ or to produce the CD $(204 \mathrm{GHz})$ required for special needed, as NTM stabilization. For CD we have assumed an upshift factor of 1.34 and the possibility to use the same diamond window, this lead to a step of $34 \mathrm{GHz}$ between the two operating modes. A second option is based on frequency step-tunable gyrotrons, capable to operate in steps of about 2 to $3 \mathrm{GHz}$ over a 10 to $12 \mathrm{GHz}$ bandwidth in order to deposit power at different resonating fields. This option can be useful if the launcher cannot direct the power in the required positions. The step tunable gyrotron will be used in association with a broadband RF output window (e.g. Brewster-angle window) described in the previous paragraph on R\&D activities.

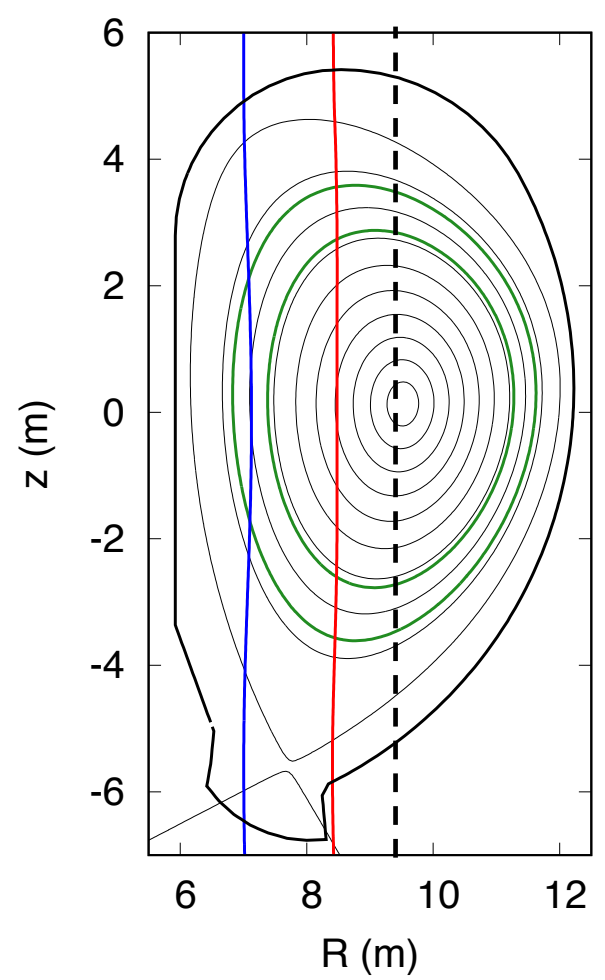

Figure 2. Section of EU DEMO plasma. Vertical lines indicate the cold resonances for $204 \mathrm{GHz}$ (blue) and $170 \mathrm{GHz}$ (red). The dashed black lines represent the shifted $204 \mathrm{GHz}$ resonance for $\mathrm{CD}$, considering an upshift of 1.34 due to a toroidal injection angle of $30^{\circ}$. The relevant $q=3 / 2$ and 2 resonant surfaces are shown in green.

Transmission Line (TL): On present experimental fusion devices two solutions for TL with a high power handling capability are adopted: Evacuated corrugated waveguide (EWG) (DIII- 
$\mathrm{D}, \mathrm{TCV}$ ) and Quasi-Optical (QO) in air as used for W7-X. These two options are under consideration starting from a preliminary study of an evacuated quasi-optical (EQO) multiplebeam TL, that can be considered for a hybrid (EWG+EQO line) solution containing the benefits of both. The main EU DEMO TL requirements are: target efficiency of $90 \%$, power handling of $2 \mathrm{MW} \mathrm{CW}$, multi-frequency (or broadband) capability and tritium compatibility; moreover it has to be considered that in DEMO the number of installed lines will be relevant (around 40 as discussed before under system reliability requirements). A Multi-Beam TL (MBTL), to transmit the power of the 8 gyrotrons of a cluster, could be a compact solution capable to reduce the complexity of the system and to save space and components provided that the distance is not excessive. As power transmission in air is not compatible with tritium segregation requirements (in case of failure in the torus window) we propose an MBTL enclosed in a vacuum vessel.

The reference design is based on mirror confocal layout with single units composed by two mirrors forming a dogleg used both for line bends (periscopes) and (replicated a few times) for transmitting along straight paths (see figure 3). One pumping system is foreseen for each unit and all mirrors are water-cooled. The characteristic length of the system $L$ is defined as the distance between the two focusing mirrors; the additional plane mirrors are foreseen mainly to straighten the beam path.

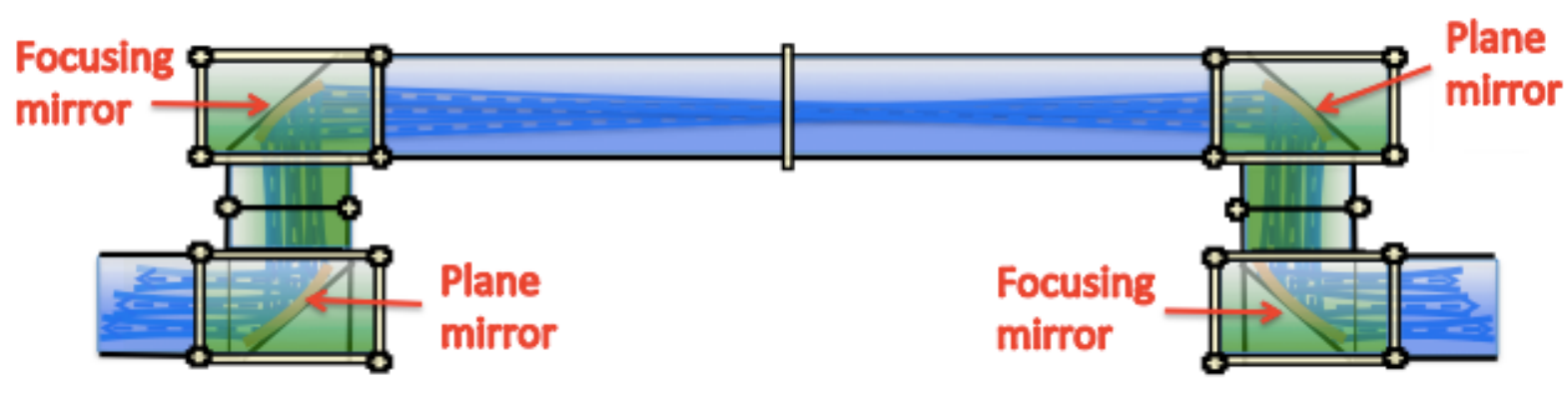

Figure 3. Sketch of the Evacuated Quasi-Optical section

In order to validate this solution a preliminary analysis has been conducted. A single circular oversized focusing mirror is considered to transmit up to 8 single overlapping beams arranged on vertices of a regular heptagon and the eighth in the center (figure 4 left). The theoretical absorbed power density on a mirror surface is function of $L$, being the diameter of mirror depending from relative distance. The minimum mirror radius has been set $r=1.75 w+90 \mathrm{~mm}$ where $w$ is the beam radius equal to $w=w_{0} \sqrt{1+\left(\frac{\lambda L}{2 \pi w_{0}^{2}}\right)}$, where $w_{0}=20.43 \mathrm{~mm}$ is the beam waist at the aperture of a waveguide of diameter $63.5 \mathrm{~mm}, \lambda$ the wavelength and $90 \mathrm{~mm}$ the distance between a vertex and the center of the heptagon (table 2).

Table 2. Minimum mirror radius for two different $L$ values. A TL length of $150 \mathrm{~m}$ is assumed.

\begin{tabular}{|c|c|c|c|}
\hline & & $\mathrm{r}=1,75 \mathrm{w}+90 \mathrm{~mm}$ & $\mathrm{r}=2 \mathrm{w}+90 \mathrm{~mm}$ \\
\hline $\mathrm{L}=8 \mathrm{~m}$ & $170 \mathrm{GHz}$ & $286 \mathrm{~mm}$ & $314 \mathrm{~mm}$ \\
\cline { 2 - 4 }$(40$ mirrors $)$ & $204 \mathrm{GHz}$ & $254 \mathrm{~mm}$ & $278 \mathrm{~mm}$ \\
\hline \multirow{2}{L}{$=12 \mathrm{~m}$} & $170 \mathrm{GHz}$ & $381 \mathrm{~mm}$ & $422 \mathrm{~mm}$ \\
\cline { 2 - 4 }$(28$ mirrors $)$ & $204 \mathrm{GHz}$ & $333 \mathrm{~mm}$ & $368 \mathrm{~mm}$ \\
\hline
\end{tabular}

Considering 8 Gaussian beams, each one of 2MW (assuming a conservative mix of $50 \%$ of either polarization), incident at $45^{\circ}$ angle on a copper surface, the absorbed power density evaluated in different mirror points at $170 / 204 \mathrm{GHz}$ is $<0.4 \mathrm{MW} / \mathrm{m}^{2}$ for $L>\sim 5 / 6.5 \mathrm{~m}$ and $<0.3 \mathrm{MW} / \mathrm{m}^{2}$ for $L>\sim 10 / 11 \mathrm{~m}$ (figure 4 right). 

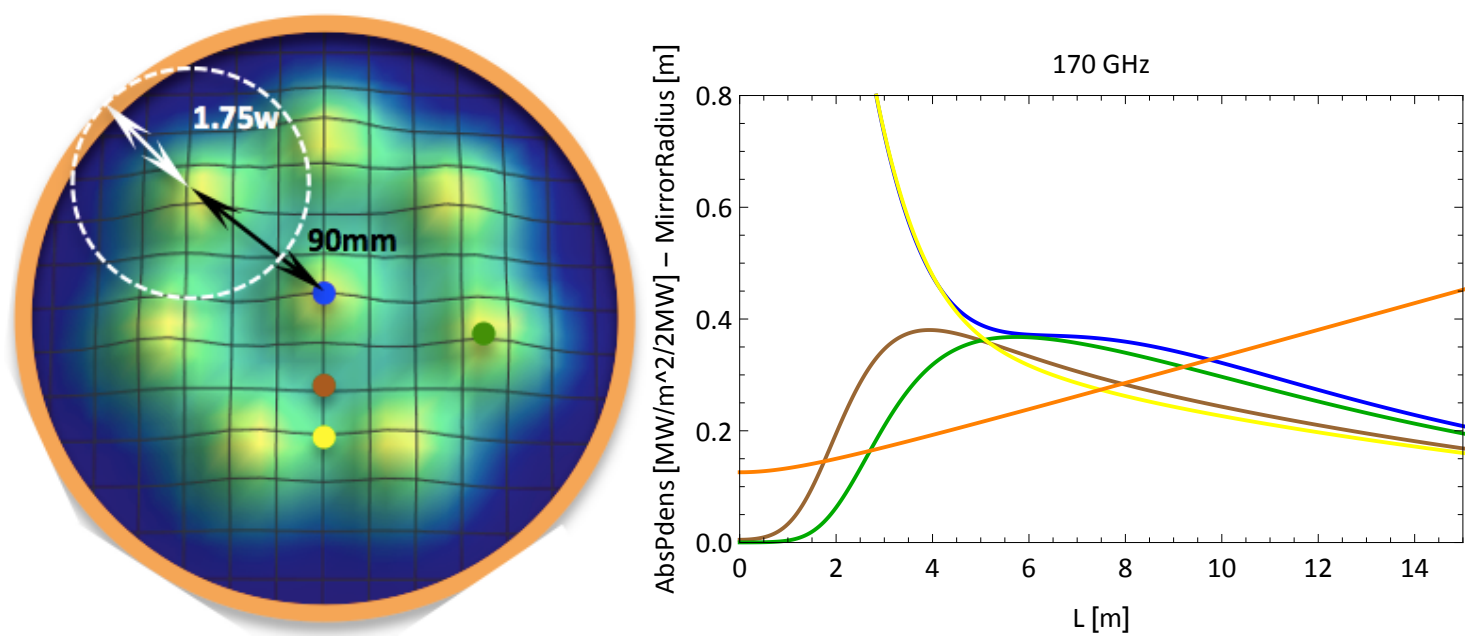

Figure 4. Left: an example of 8 beams disposed on a mirror surface with the beam envelope (orange circle). Right: Absorbed power density in function of $L$ for different points of the mirror surface (see left) at $170 \mathrm{GHz}$. In orange the beam envelope radius.

An overall estimation of losses has been carried out for a generic EQO with 150m length and $L=8 / 12 \mathrm{~m}$ for $170 \mathrm{GHz}$ and $204 \mathrm{GHz}$. Table 3 shows theoretical ohmic losses estimation for a TL of several ideal surface copper mirrors with $90^{\circ}$ bends: the incident wave polarization can be chosen appropriately (if the bends are kept in one plane along the whole TL path) to halve the losses (saving 3-7\% of 50MW means sparing 1-2 gyrotrons). The contribution of polarizers is not included [34]. A second main source of loss is the beam truncation reported in table 4 for two different MBTL mirror radius. Equivalent values have to be foreseen for mode conversion losses. The misalignment effect contribution has been rescaled with the TL length on the basis of W7-X QO TL [35]; for the $150 \mathrm{~m}$ of TL is $\sim 3 \%$. The transmission efficiency for $\mathrm{L}=8 \mathrm{~m}$ (worst case) at $170 \mathrm{GHz}$ is in the range $88 \%-91 \%$, close to the initial DEMO requirement $(90 \%)$ and to the EWG estimated efficiency $(88-90 \%$ at $170 \mathrm{GHz}$, depending of the number of mitre bends). The eventual losses due to the presence of the enveloping vacuum-vessel wall and the mirror surface deformation have not been considered yet and will be subject of future dedicated studies.

Table 3. Ohmic losses estimation of DEMO EQO transmission losses for $\sim 150 \mathrm{~m}$ of TL with 40 mirrors.

\begin{tabular}{|l|l|l|l|l|}
\hline & $\begin{array}{l}\mathrm{L}=8 \mathrm{~m} \\
(40 \text { mirrors })\end{array}$ & $\begin{array}{l}\mathrm{L}=12 \mathrm{~m} \\
(28 \text { mirrors })\end{array}$ & $\begin{array}{l}\mathrm{L}=8 \mathrm{~m} \\
(40 \text { mirrors })\end{array}$ & $\begin{array}{l}\mathrm{L}=12 \mathrm{~m} \\
\text { (28 mirrors) }\end{array}$ \\
\hline Absorption on mirrors: & $\begin{array}{l}170 \mathrm{GHz} \\
\text { loss }[\%]\end{array}$ & $\begin{array}{l}170 \mathrm{GHz} \\
\text { loss }[\%]\end{array}$ & $\begin{array}{l}204 \mathrm{GHz} \\
\text { loss }[\%]\end{array}$ & $\begin{array}{l}204 \mathrm{GHz} \\
\text { loss }[\%]\end{array}$ \\
\hline H-Plane, Inc. angle $45^{\circ}$ & $3,18 \%$ & $2,23 \%$ & $3,47 \%$ & $2,45 \%$ \\
E-Plane, Inc. angle 45 & $6,26 \%$ & $4,42 \%$ & $6,83 \%$ & $4.83 \%$ \\
\hline
\end{tabular}

Table 4. Truncation losses estimation of $\sim 150 \mathrm{~m}$ DEMO EQO TL evaluated at two different distances $L$ and three mirror radius.

\begin{tabular}{|c|c|c|c|}
\hline & & $\mathrm{r}=1,75 \mathrm{w}+90 \mathrm{~mm}$ & $\mathrm{r}=2 \mathrm{w}+90 \mathrm{~mm}$ \\
\hline $\mathrm{L}=8 \mathrm{~m}$ & $170 \mathrm{GHz}$ & $1,50 \%$ & $0,21 \%$ \\
\hline
\end{tabular}




\begin{tabular}{|c|c|c|c|}
\hline (40 mirrors) & $204 \mathrm{GHz}$ & $1,41 \%$ & $0,20 \%$ \\
\hline $\mathrm{L}=12 \mathrm{~m}$ & $170 \mathrm{GHz}$ & $1,24 \%$ & $0,18 \%$ \\
\cline { 2 - 4 }$(28$ mirrors $)$ & $204 \mathrm{GHz}$ & $1,14 \%$ & $0,16 \%$ \\
\hline
\end{tabular}

A preliminary cost analysis has been carried out considering three main contributions: mirrors, vacuum envelope and pumping system. The latter two are based on recent quotations provided by companies expert in the field whereas the mirror cost is taken from W7-X [36] rescaled with dimensions. The cost estimation per meter as a function of $L, r=1.75 w+90 \mathrm{~mm}$ normalized to the EWGs cost for 2009 ECRH4JET project $[37,38]$ is $<0.6$ for $\mathrm{L}>2 \mathrm{~m}$. After $\sim 8 \mathrm{~m}$ the cost reaches a lower saturation $(<0.4)$ because the reduction of number of mirrors is counterbalanced by the mirror size increase. The increase for a larger radius $(r=2 w+90 \mathrm{~mm})$ is $\sim 10-15 \%$.

Antennas: DEMO EC launching system is composed of launchers with a sufficient flexibility and without movable parts in the proximity of the plasma. Remote Steering Antennas (RSAs) or simple truncated waveguides, in conjunction with step tunable gyrotrons, are candidates for such a launcher. An extensive engineering effort to integrate the antennas into the tokamak is presently carried on and reported in [39,40]. Two concepts are under assessment: a Blanket Separated Design (BSD), where the port plug stops behind the Outboard Multi-Module Segment (OB-MMS) and a Blanket Integrated Design (BID), where the port plug penetrates the OB-MMS up to the plasma. A general assessment, based on EU DEMO 2015 baseline design and on beam tracing code TORBEAM [41], has been performed to evaluate launching performance and plasma accessibility from a RSA located in different points situated in a poloidal section and scanning different frequencies (from $140 \mathrm{GHz}$ to $230 \mathrm{GHz}$, with step of $10 \mathrm{GHz})$. For each frequency the steering angle has been varied $\left( \pm 15^{\circ}\right)$ in order to reach different radial position. The plane of steering has been also chosen to exploit the widest range of possibilities. Results in terms of deposition location accessibility $\rho$ (normalized plasma radius) and driven current $I_{C D}$ are shown in figure 5 when a beam $(170 \mathrm{GHz}$ on the left, $200 \mathrm{GHz}$ the closest value to $204 \mathrm{GHz}$ in the performed scan, on the right, $w_{0}=20.4 \mathrm{~mm}$ ) is launched from an equatorial port plug point (EPP3, $\mathrm{R}=13.545 \mathrm{~m}$ and $\mathrm{Z}=0 \mathrm{~m}$ ) with toroidal and poloidal injection angles in the range $\beta=\left[0^{\circ}, 30^{\circ}\right]$ and $\alpha=\left[-30^{\circ}, 30^{\circ}\right]$, respectively. Figure 6 shows the results for the same launching point EPP3 when different frequencies are considered for a given steering plane, centred on $\alpha_{0}=-20^{\circ}, \beta_{0}=26^{\circ}$ and properly oriented. In this case the largest plasma coverage is obtained with the higher frequency showing a sufficient CD efficiency also for outer radii, as the requirement for NTM stabilization is to drive current up to $\rho=0.85$. These studies shown as the localizations required in table 1 can be reached with the two frequencies selected, even if it is necessary a deeper analysis to evaluate the power deposition once the launching point will be defined. The reduced deposition range for lower frequency in figure 6 is due to the limited steering range of and RSA for the selected steering plane. To increase the ECCD efficiency it is necessary explore the possibility of higher poloidally launching points with different steering plane orientations at high enough frequency. 


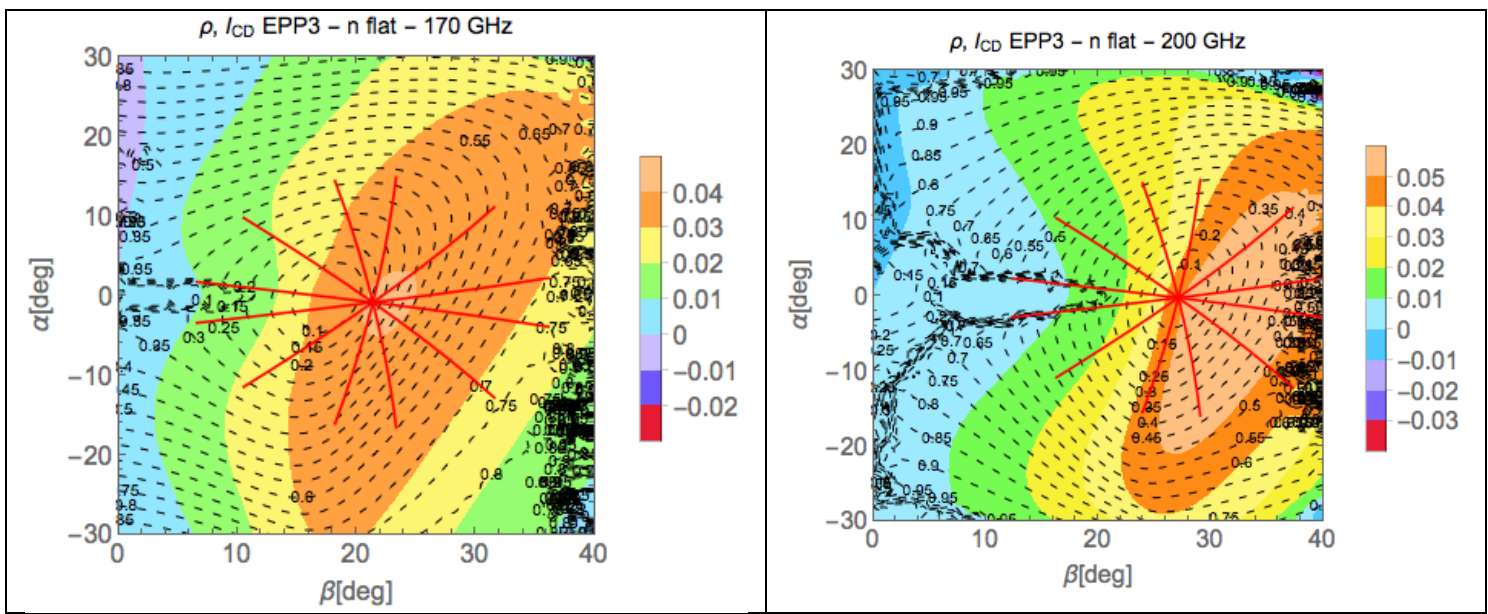

Figure 5. Contour plots for normalized deposition location $\rho$ (black dashed curves) and total driven current $I_{C D}$ (color code, $M A / M W$ ) as a function of the injection angles $(\alpha, \beta)$ with different possible steering planes (red lines) in the parameters space. The cases of $170 \mathrm{GHz}$ frequency (left) and 200GHz (right) are shown.

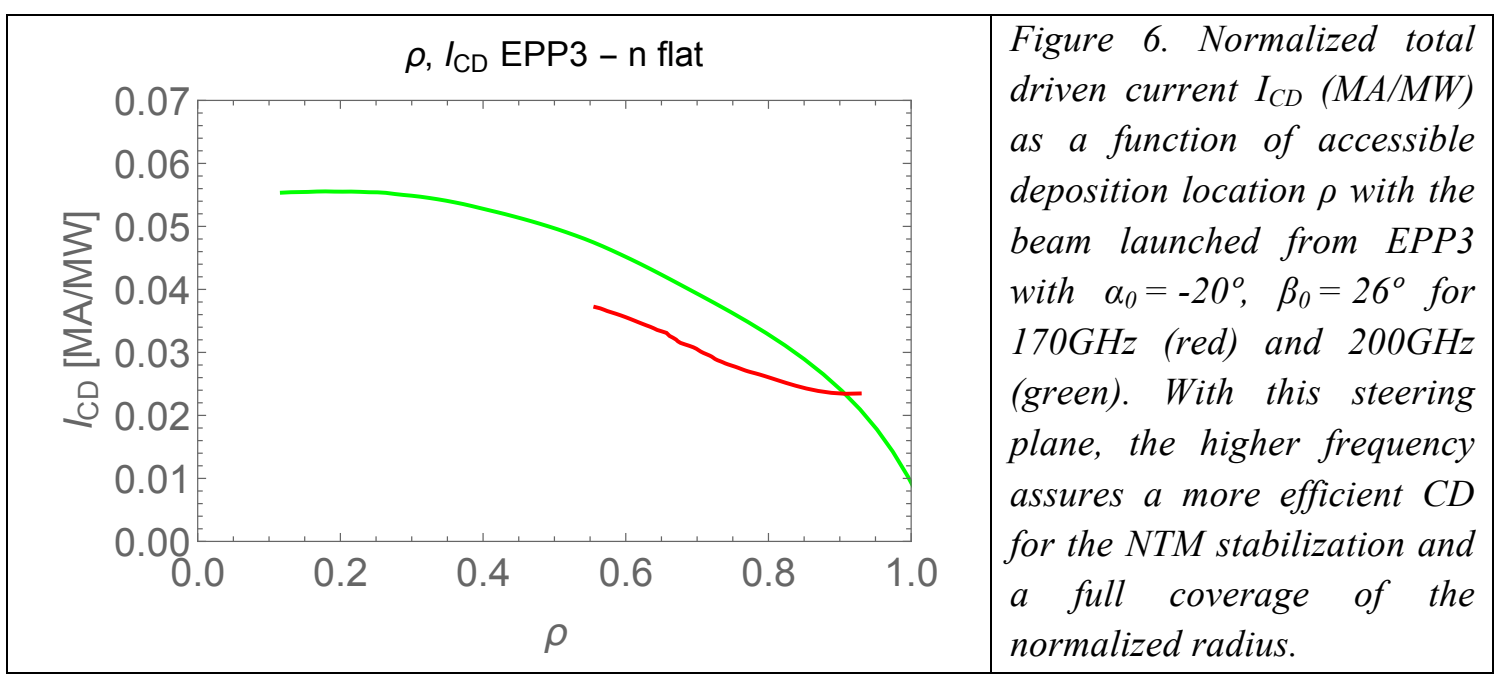

A preliminary estimation of the minimum apertures required by the launchers on the Breeding Blanket (BB) has been done in order to allow a first evaluation of the impact on TBR of the EC launchers. Calculations $[39,40]$ results in $\triangle \mathrm{TBR}$ of $\sim 0.022$ for $50 \mathrm{MW}$ of injected power launched through 5 equatorial ports. As said, this value is obtained considering a conservative approach and can be reduced as the design will improve.

\section{Conclusion}

The status of activities on the EC DEMO system in the framework of Work Package Heating and Current Drive under the umbrella of EUROfusion consortium are presented and discussed. The activities in the frame of gyrotron R\&D and Advanced Developments (up to $240 \mathrm{GHz}, 2 \mathrm{MW}$ RF output power, $>60 \%$ efficiency, multi-purpose and step-frequency tunable operation) are considered strategically necessary in order to prepare mature technology not only for the pulsed EU DEMO1 but also for the steady state EU DEMO2 device. Major parts in the research are focusing on the coaxial-cavity gyrotron technology, manufacturing and 
implementation of large CVD diamond-disc Brewster-angle windows and multi-stage depressed collectors.

A conceptual design of EC system starting on the physical requirements for EU DEMO1 and on the constraints for a nuclear power plant is also presented. An architecture based on 5 identical clusters of 8 gyrotrons connected with a multi-beam transmission line to 8 singular remote steering antennas is discussed and motivated on the basis of technical requirements and RAMI consideration.

\section{Acknowledgment}

This work has been carried out within the framework of the EUROfusion Consortium and has received funding from the EURATOM research and training programme 2014-2018 under grant agreement No 633053. The views and opinions expressed herein do not necessarily reflect those of the European Commission.

\section{References}

[1] M. Q. Tran et al. EU DEMO Heating and Current Drive: Physics and Technology 26th FEC - Kyoto (2016) FIP/7-7

[2] A. Bader et al. Ion Cyclotron Range of Frequency Power for DEMO, 26th FEC - Kyoto (2016) FIP/P7-13

[3] P. Sonato et al., Nucl. Fusion 57, 056026 (2017)

[4] P. Vincenzi et al., Fusion Eng. Des., In press (2017)

[doi:10.1016/j.fusengdes.2017.02.064]

[5] G. Giruzzi, et al., Nucl. Fusion 55, 073002 (2015)

[6] R. Wenninger et al., Nucl. Fusion 55, 063003 (2015)

[7] G. Federici et al., Fus. Eng. Des. 89, 882-889 (2014)

[8] H. Zohm et al., "A Stepladder Approach to a Tokamak Fusion Power Plant" Nucl. Fusion submitted for publication (2017)

[9] E. Poli et al., Nucl. Fusion 53, 013011 (2013)

[10] B. Piosczyk et al., IEEE Trans. Plasma Science, 32, 3, 413-417 (2004)

[11] J. Franck et al., Nucl. Fusion 55, 013005 (2015)

[12] J. Franck et al., IEEE Trans. Electron Devices, 63, 9, 3740-3746 (2016)

[13] T. Rzesnicki et al., IEEE Trans. Plasma Science, 38, 6, 1141-1149 (2010)

[14] T. Rzesnicki et al., Proc. Int. Conf. on IRMMW-THz, W4/D-25.10 (2014)

[15] I. Gr. Pagonakis et al., Physics of Plasmas, 23, 023150 (2016)

[16] I. Gr. Pagonakis et al., Physics of Plasmas, 23, 083103 (2016)

[17] S. Ruess et al., IEEE Trans. Electron Devices, 63, 5, 2104-2109 (2016)

[18] P. Kalaria et al., Phys. Plasmas 23, 092503 (2016)

[19] K. A. Avramides et al., EPJ Web of Conferences, 32, 04016 (2012)

[20] P. Kalaria et al., IEEE Proc. of 2016 German Microwave Conference (GeMiC), Bochum, 2016, pp. 377-380. [doi: 10.1109/GEMIC.2016.7461635]

[21] M. Thumm et al., Fusion Eng. Des., 53, 1, 517-524 (2001)

[22] G. Gantenbein et al., IEEE Transactions on Electron Devices, 61, 6, 1806-1811 (2014)

[23] A. Samartsev et al., IEEE Trans. Electron Devices, 62, 7 (2015)

[24] A. Singh et al., IEEE Trans. Plasma Science, 27, 2, 490-502 (1999)

[25] R.L. Ives et al., IEEE Trans Plasma Science, 27, 2, 503-511 (1999)

[26] G. P. Saraph et al, IEEE Trans. Plasma Science, 28, 3, 830-840 (2000)

[27] G. Ling et al., IEEE Trans. Plasma Science, 28, 3, 606-612 (2000)

[28] I. Gr. Pagonakis et al., IEEE Trans. Plasma Science, 36, 2 (2008)

[29] I. Gr. Pagonakis et al., Physics of Plasmas, 23, 043114 (2016) 
[30] C. Wu et al. Physics of Plasmas, 24, 043102 (2017)

[31] Institute of Electrical and Electronics Engineers (1990) IEEE Standard Computer Dictionary: A Compilation of IEEE Standard Computer Glossaries. New York, NY ISBN 1-55937-079-3

[32] T. Franke et al., Fusion Eng. Des., In press (2017) [doi:10.1016/j.fusengdes.2017.02.007]

[33] S. Garavaglia et al., EPJ Web of Conferences, In Press (2017)

[34] V. Erckmann et al. Fusion Sci. Technol. 55, 16-22 (2009)

[35] V. Erckmann et al. Fusion Sci. Technol. 52, 291-312 (2006)

[36] W. Kasparek, private communication (2009)

[37] G. Giruzzi, et al., Nucl. Fusion 51, 063033 (2011)

[38] http://users.euro-fusion.org/pages/ecrh/E4J_2009_Report.pdf

[39] S. Garavaglia et al., AIP Conf. Proc. 1689, 090009 (2015)

[40] G. Grossetti et al. DEMO Port Plug Design and Integration Studies. 26th FEC - Kyoto (2016) FIP/P7-4

[41] E. Poli et al., Computer Physics Communications, 136, 1-2, 90-104 (2001) 